\title{
Prediction of the Moving Direction of Google Inc. Stock Price Using Support Vector Classification and Regression
}

\author{
Liu Pan \\ Department of Business English, Gannan Normal University \\ Economic \& Technological Development Zone, Ganzhou 341000, China \\ E-mail:panliu18@gmail.com
}

\begin{abstract}
Xuan Liu (Corresponding author)
Department of Electrical and Computer Engineering, Johns Hopkins University

3400 N. Charles St, Baltimore, MD, USA.
\end{abstract}

Tel: 1-90-6487-1634 E-mail: xuliu@mtu.edu

Received: April 16, 2014 Accepted: May 18, $2014 \quad$ Published: June 1, 2014

doi:10.5296/ajfa.v6i1.5485 URL: http://dx.doi.org/10.5296/ajfa.v6i1.5485

\begin{abstract}
Forecasting the short-term trend of a stock market has long been a big challenging task. Parameters of stock markets, including open/close prices, daily-high/low prices and trading volumes, were frequently used in previous studies to forecast the stock market. Basing on the fact that the moving direction of these parameters have certain inertia within short-term period, we here explored the potential application of the moving trends of these parameters within 4 different time periods $(5,15,30$ and 45 trading days respectively) for forecasting the movement direction of stock price of Google Inc. by using support vector classification (SVC) and support vector regression (SVR). We found that among the 4 different time periods tested, the moving trend within 30 days has the best accuracy on the prediction of the stock price of Google Inc., and using SVC and SVR combination improved the prediction performance. These results indicated that moving trends of stock transaction data within a certain time period have good inertia and are thus useful for forecasting the moving direction of stock price.
\end{abstract}


Keywords: Financial forecasting, Stock price prediction, Trend prediction, Technical analysis, Support vector machine, Support vector classification, Support vector regression 


\section{Introduction}

Analysis of stock market, including the stock price forecasting, has long been an intriguing topic for both investigators and researchers. Fundamental analysis and technical analysis are the two main stock analysis strategies used to forecast the future stock price movements (Murphy, 1999; Turner, 2007). Fundamental analysis attempts to predict the price of a particular stock by studying the company fundamentals such as revenues, annual growth rates, and potential competitors (Murphy, 1999). Technical analysis, on the other hand, is solely based on the study of stock market's historical data or pattern, including price, volume action and technical indicators (Turner, 2007). Both approaches have their own Pros and Cons. Generally, fundamental analysis is favored for longer time frames, while technical analysis is considered as a better style for short-term trading.

Forecasting the short-term trend of a stock market is still a big challenging task nowadays. This is because that stock market is noisy, chaotic, nonparametric and non-linear in nature, and many external entities like politics, human psychology/behavior, liquid money and related news influence the direction of the stock market (Abu-Mostafa and Atiya, 1996). Recently, a lot of interesting work has been carrying on in the area of applying machine learning algorithms, including support vector machine (SVM), for analyzing price patterns and predicting stock prices and index changes (Yang et al., 2002; Grosan and Abraham, 2006; Chen et al., 2006; Sapankevych and Sankar, 2009; Kao et al., 2013; Kazem et al., 2013; Zhi-gang et al., 2013). The advantage of SVM is that it is able to reach the global optimum and is resistant to the undertraining or overtraining problems (Yoo et al., 2005; Chen et al., 2006; Sapankevych and Sankar, 2009). This machine learning method has been successfully used for stock return predictions in several financial areas (Yang et al., 2002; Chen et al., 2006; Sapankevych and Sankar, 2009; Kao et al., 2013; Kazem et al., 2013).

Parameters of stock markets, including open/close prices, daily-high/low prices and trading volumes, were frequently used in previous studies to forecast the stock market (Lildholdt, 2002; Fiess and MacDonald, 2002; Corwin and Schultz, 2012; Fuertes and Olmo, 2013). Considering that the moving trends of these parameters may have certain inertia within short-term period, we hypothesized that the moving direction of these parameters would be better than the original parameters themselves for predicting the short-term stock price. To test this hypothesis, in this study we explored the potential application of the moving trends of these stock parameters to forecast the movement direction of stock price of Google Inc. by using support vector classification (SVC) and support vector regression (SVR), two main SVM application forms.

The remaining sections of this report are organized as following: section 2 provides a brief overview of the SVM algorithms; Section 3 describes the experiment design; Section 4 reports and discusses the experiment results; Section 5 summarizes the whole report.

\section{Methodology}

\subsection{The basic ideas of SVC and SVR}

SVMs, a set of supervised learning algorithms developed by Vapnik and his co-workers, are characterized by usage of kernels, absence of local minima, sparseness of the solution and 


\section{Macrothink}

capacity control obtained by acting on the margin or on number of support vectors (Vapnik, 1995). According to the purposes, SVMs can be divided into SVC, SVR, and ranking SVM. SVC performs classification by finding the hyperplane that maximizes the margin between the two classes in high- or infinite-dimensional space. SVR is extended from SVM and performs regression in the high-dimension feature space using insensitive loss. Both SVC and SVR are widely used in various areas and continue to be two of the most successful machine learning algorithms. An overview of the basic ideas of SVC and SVR was described in detail in reference (Yoo et al., 2005). Briefly, for a two-class classification problem, we can assume that we have a set of input data points $x_{i} \in \mathrm{R}^{\mathrm{d}}(i=1,2, \ldots, N)$ along with each point's classification $y_{i}$, where $y_{i}$ can take on one of two possible values: -1 or 1 . The linear support vector machine is defined as the following optimization problem:

$$
\begin{aligned}
& \min \frac{1}{2} \mathrm{w}^{T} \cdot \mathrm{w}+\underset{i=1}{C \sum_{\mathrm{w}}} \xi_{i} \\
& \text { s.t. } y_{i}\left(\mathrm{w}^{t} \cdot \mathrm{x}_{i}+b\right) \geq 1-\xi, \xi_{i} \geq 0 i=1 \ldots l
\end{aligned}
$$

where $\xi_{i}$ is the error for a given training point $x_{i}, w$ is the margin, $b$ is the offset for the hyperplane, and $C$ is a constant representing the emphasis that is to be placed on minimizing the error. The solution to this problem results in the following classifier for the prediction of $\mathrm{f}(\mathrm{x})$ in the new sample $\mathrm{x}$ :

$$
f(x)=\operatorname{sign}\left(\sum_{i=1}^{l} y_{i} \alpha_{i} \cdot K\left(x, x_{i}\right)+b\right)
$$

where $\alpha_{l}$ is the parameter coefficient, and $\mathrm{K}\left(\mathrm{x}, \mathrm{x}_{i}\right)$ is kernel function.

For SVR, it is formulated as minimization of the following functional:

$$
\begin{aligned}
& \min _{\mathrm{w}, b} \frac{1}{2} \mathrm{w}^{T} \cdot \mathrm{w}+\mathrm{C} \sum_{i=1}\left(\xi_{i}+\xi_{i}^{*}\right) \\
& \int_{\text {s.t. }\{} y_{i}-\left(\mathrm{w}^{T} \cdot \mathrm{w}^{T} \cdot x_{i}+b\right) \leq \varepsilon+\xi_{i}
\end{aligned}
$$

Where $\varepsilon$ is the parameter epsilon and the couple (xi, yi) the training Set. Slack variables $\xi$ and $\xi^{*}$ were used to allow some errors to deal with noise in the training data. Once trained, the SVR will generate predictions using the following formula: 


$$
f(x) \equiv \sum_{i=1}^{l}\left(\alpha_{l}-\alpha_{i}^{*}\right) \cdot K\left(x, x_{i}\right)+b
$$

\subsection{Kernel and optimization of kernel parameters}

Epsilon-SVR in LIBSVM package, which is developed by Chang et al (2011) and is currently one of the most widely used SVM, was employed for stock price prediction in this study. LIBSVM supports four basic kernel functions (Hsu et al., 2005; Chang and Lin, 2011):

(1) Linear Kernel: $K(\mathrm{x}, \mathrm{y})=\mathrm{x} \cdot \mathrm{y}$

(2) Polynomial Kernel: $K(\mathrm{x}, \mathrm{y})=(\gamma \cdot \mathrm{x} \cdot \mathrm{y}+\operatorname{coef} 0)^{\text {degree }}$

(2) Radial Basis function $(R B F)$ Kernel: $K(\mathrm{x}, \mathrm{y})=\exp \left(-\gamma \cdot\|\mathrm{x}-\mathrm{y}\|^{2}\right)$

(3) Sigmoid Kernel: $K(\mathrm{x}, \mathrm{y})=\tanh (\gamma \cdot \mathrm{x} \cdot \mathrm{y}+\operatorname{coef} 0)$

We chose RBF Kernel in our study because of the following factors: 1) RBF kernel has fewer numerical difficulties and can handle the nonlinear models (Hsu et al., 2005); 2) The linear kernel can be regard as a special case of RBF Kernel; 3) The polynomial kernel has more hyperparameters than the RBF kernel; 4) The sigmoid kernel behaves like RBF for certain parameters (Hsu et al., 2005).

The SVM performance depends on a good setting of two parameters C and $\gamma$ (Hsu et al., 2005; Chang and Lin, 2011). Basing on 3-fold cross-validation error, we obtained the best values of parameters $C$ and $\gamma$ for SVC and SVR using grid search method.

\section{Experiment Design}

We chose the internet search giant Google Inc (stock symbol: GOOG) for this study basing on two reasons: 1) Its transaction data is simpler than other stocks since this company has no divide-paying and stock-splitting/combining records; 2) The company has very high volume of outstanding shares and high stock price, making it difficult for the stock price being manipulated. We obtained the transaction data and historical prices of Google Inc. from the date of its initial public offering (Aug 19, 2004) to Dec 31, 2013 from Yahoo Finance (http://finance.yahoo.com). The original dataset contains 6 attributes: date, open price, high price, low price, close price, volumes and adjusted close price. The adjusted close prices are the same as the close price of Google Inc. as the company has never paid divides and the its stock shares have never been split or combined. Figure 1 shows the overall moving trend of the close prices for Google Inc. traded on the NASDAQ exchange from 2004 to 2013. 


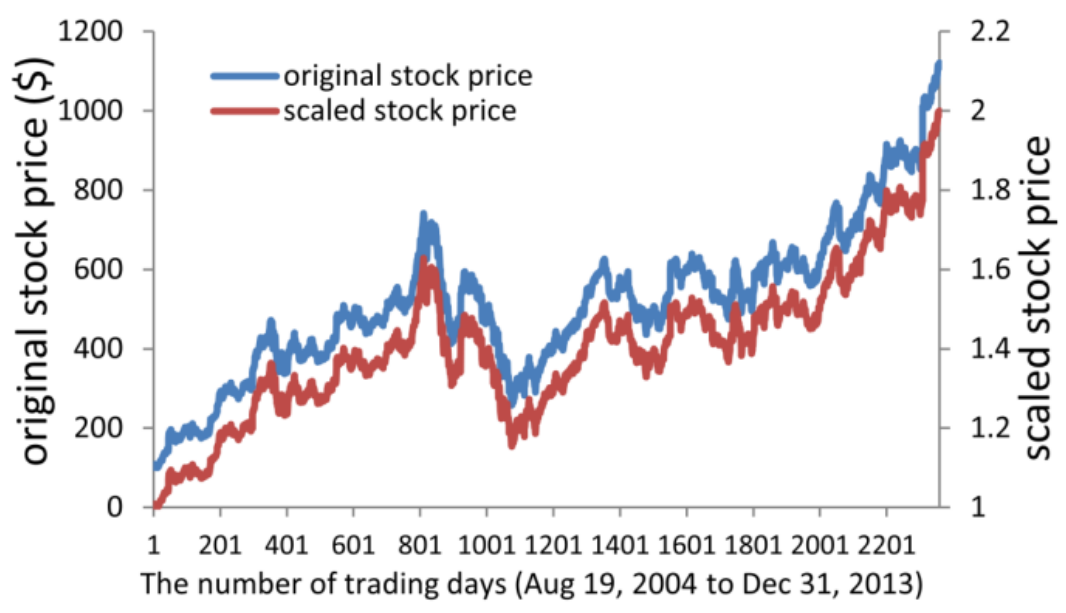

Figure 1. The overall moving trend of original (left y-axis) and scaled (right y-axis) close price for Google Inc. for the year of 2004 to 2013

To examine within which time period the moving trend will be better for prediction of the stock direction, in this study we analyzed the prediction accuracy of the moving trends of GOOG transaction data within 4 different time periods, including periods of 5, 15, 30 and 45 trading days, respectively. The SVM classifiers generated basing on the 4 moving trends are named correspondingly as classifiers $1,2,3$ and 4 , respectively. The moving trend of the transaction data within a time period is defined as the slope (value b) in the regression formula $\mathrm{Y}=\mathrm{a}+\mathrm{bX}$ that is produced by simple linear regression analysis, in which $\mathrm{X}$ is the number of trading day (from 1 to 5 for classifier 1,1 to 15 for classifier 2, 1 to 30 for classifier 3 and 1 to 45 for classifier 4), and $\mathrm{Y}$ is the corresponding data (open price, high price, low price, close price or volume) at the corresponding trading date. The obtained slopes of the open prices, high prices, low prices, close prices and volumes of GOOG within 5 (classifier 1), 15 (classifier 2), 30 (classifier 3) or 45 (classifier 4) training days were then used as five input features for SVM to predict the slopes of close price of GOOG in the next $5^{\text {th }}, 15^{\text {th }}, 30^{\text {th }}$ or $45^{\text {th }}$ days, respectively. The predicted slope of GOOG close price $>0$ represents the stock price rose up during that period, while slope $<0$ means price falling down.

To check the prediction accuracy, the predicted results were compared with the actual data (called indicator). The slope values of the close price of GOOG were directly used as indicators in SVR analysis. In SVC analysis, a Boolean value (1 or -1) that was transformed from the slope values of the close price was used as indicators. Boolean value ' 1 ' means the corresponding slope value $>0$, while ' -1 ' means the corresponding slope $<0$.

All the datasets were split into two parts for both SVC and SVR prediction in this study: training set (accounting for about $2 / 3$ of the data) and test set (the rest $1 / 3$ of the data). As large attribute values might cause numerical problems and greater numeric ranges dominating those in smaller numeric ranges (Hsu et al., 2005), we scaled all the data to the range from 1 
to 2 using Mapminmax function of Matlab before applying SVM analysis. Figure 1 shows that scaled close prices have the exactly same pattern as that of the original close prices.

The best values for parameter $\mathrm{C}$ and $\gamma$ that were obtained by grid search method for each of the SVR perdition classifier are shown in Table 1.

Table 1. SVM parameters and prediction accuracies for different classifiers tested

\begin{tabular}{|c|c|c|c|c|c|c|}
\hline \multirow[b]{2}{*}{ Classifier } & \multirow[b]{2}{*}{ Trading days } & \multirow[b]{2}{*}{ SVM } & \multirow[b]{2}{*}{ Best C } & \multirow[b]{2}{*}{ Best $\gamma$} & \multicolumn{2}{|l|}{ Predicted accuracy } \\
\hline & & & & & Training set Test se & \\
\hline \multirow[t]{2}{*}{1} & 5 & SVC & 2 & 16 & $64.33 \%(193 / 300)$ & $50.59 \%(86 / 170)$ \\
\hline & & SVR & 2 & 8 & $65.67 \%(197 / 300)$ & $50.59 \%(86 / 170)$ \\
\hline \multirow[t]{2}{*}{2} & 15 & SVC & 0.031 & 0.031 & $62.00 \%(62 / 100)$ & $53.57 \%(30 / 56)$ \\
\hline & & SVR & 0.313 & 1 & $61.00 \%(61 / 100)$ & $48.21 \%(27 / 56)$ \\
\hline \multirow[t]{2}{*}{3} & 30 & SVC & 90.510 & 0.707 & $74.00 \%(37 / 50)$ & $62.96 \%(17 / 27)$ \\
\hline & & SVR & 12.126 & 0.218 & $68.00 \%(34 / 50)$ & $59.26 \%(16 / 27)$ \\
\hline \multirow[t]{2}{*}{4} & 45 & SVC & 5.657 & 32 & $93.33 \%(28 / 30)$ & $42.86 \%(9 / 21)$ \\
\hline & & SVR & 1 & 5.278 & $70.00 \%(21 / 30)$ & $52.83 \%(11 / 21)$ \\
\hline
\end{tabular}

\section{Results and Discussions}

\subsection{Prediction of moving direction of GOOG price using SVC}

SVC analysis gave out a probability value ranging from 0 to 1 for each instance (e.g. moving direction in one trading period). The probability value closing to 1 means that the chance for GOOG stock price rising up is very high, closing to 0 means the great chance of price falling down, while close to 0.5 represents the chance of price rising-up is similar to that of falling down. Figure 2 shows the correlation between the predicted probability values and actual moving trend (slope) across all the instances analyzed. Among the 4 classifiers, the predicted pattern in classifier 3 (slopes within 30 trading days) is the most closest to the actual moving trend and has the highest Pearson's correlation coefficients (Pearson's r) in both training and test datasets. 

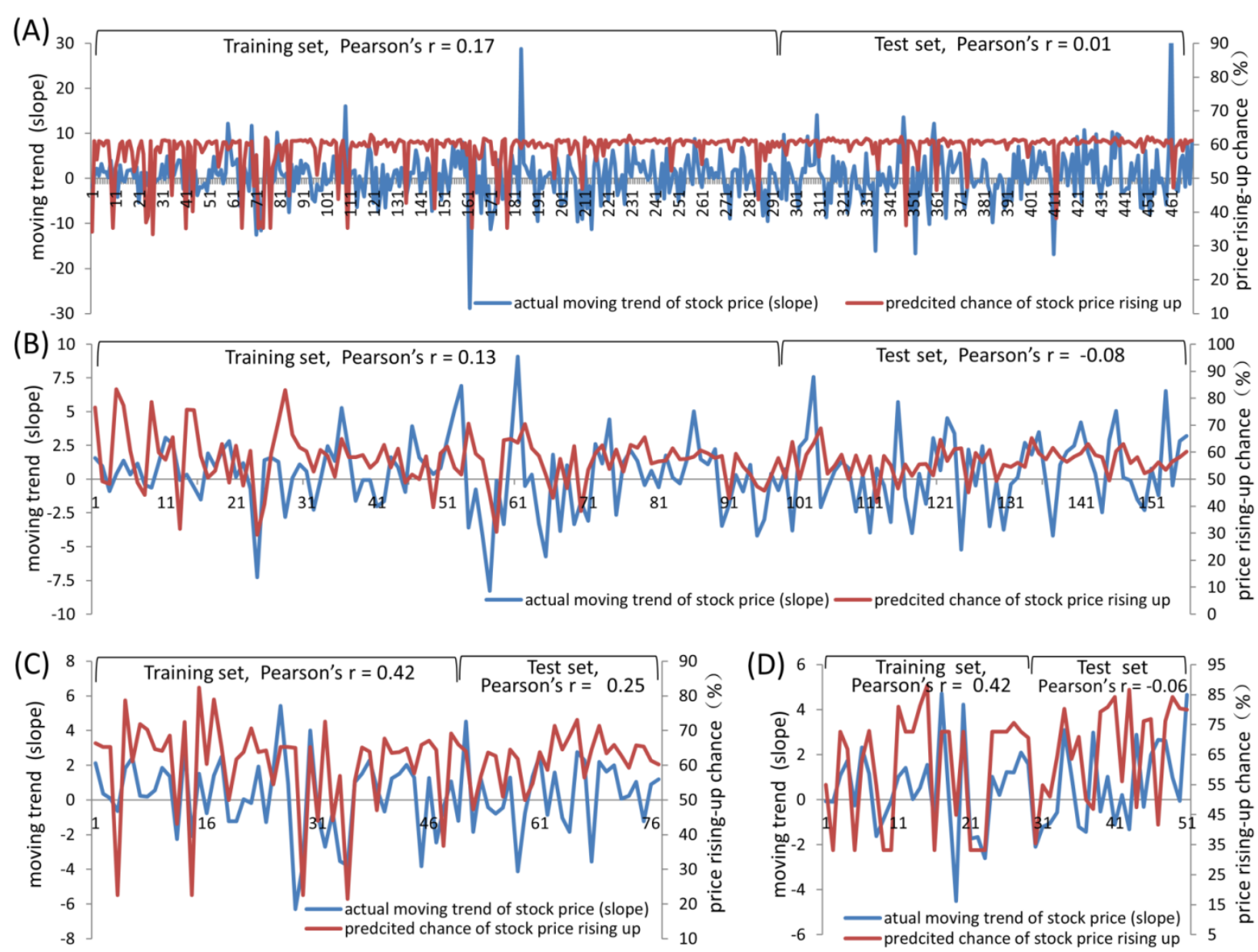

Figure 2. The correlation between the predicted possibility values and actual moving trend (slope) across all the instances (moving direction in one trading period) analyzed by SVC. (A) Classifier 1 (5d). (B) Classifier 2 (15d). (C) Classifier 3 (30d). (D) Classifier 4 (45d).

The SVC classifier automatically classified the instances with a probability value $>0.5$ into the group with price rising up (e.g. slope $>0$ ), while the rest into the group with price falling down (slope $<0$ ). The prediction accuracies of SVC analysis for different classifiers are listed in table 1. Among the 4 classifiers tested, SVC analysis using classifier 3 achieved the highest accuracy $(62.96 \%)$ for the test dataset. This number means that if we use this classifier to guide our investment in GOOG stock, we will get gains for 63 times while get losses for 37 in 100 trading times. Theoretically, this gain/loss ratio will bring about considerable returns in short-term stock trading. The prediction accuracies for the other 3 classifiers are all close to $50 \%$ for test dataset and therefore are not practical in stock trading.

When we used more stringent cut-off values for the SVC classifiers to make classification, we obtained higher prediction accuracies for the classifiers 2 and 3, especially the latter (Table 2). For example, the accuracy is $100 \%$ for the test dataset if instances with probability values $>0.7$ are classified into the price-rising up group and instances with values $<0.4$ into the price-falling down group for classifier 3 (Figure 3). 


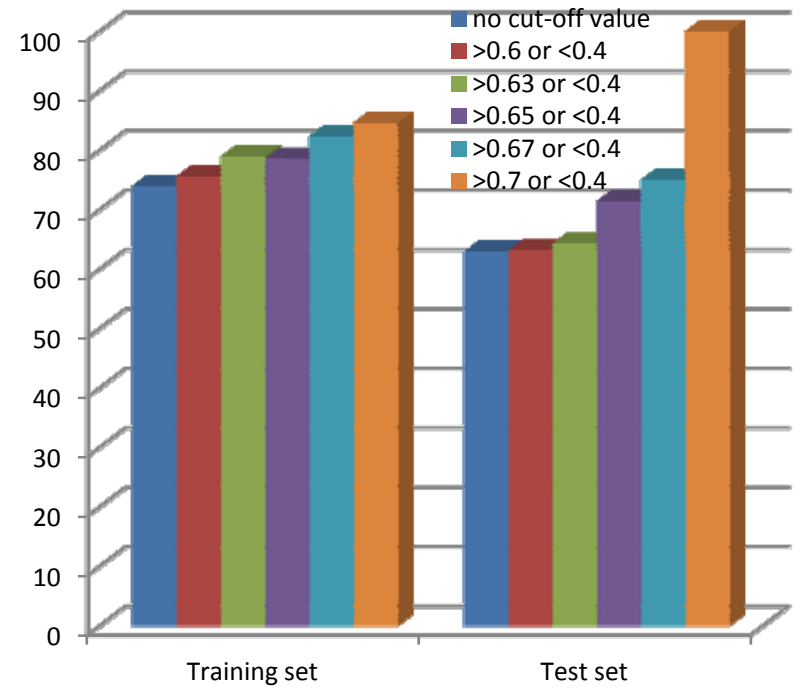

Figure 3. The Prediction accuracies when using more stringent cut-off values for the SVC classifier 3 to make classification

\subsection{Prediction of moving direction of GOOG price using SVR}

SVR analysis gave out a predicted slope value for each instance. Figure 4 shows the correlation between the predicted and actual slope values across all the instances analyzed. Like the SVC results, the predicted slope pattern in classifier 3 is the most closing to the actual pattern and has the highest Pearson's $r$ in both the training and test datasets. While for the other 3 classifiers, the patterns between predicted and actual slopes are similar only in the training dataset.

If we only consider whether the predicted moving direction by SVR is the same as the actual one (e.g., the predicted and actual slopes are both $>0$ or $<0$ ), we can also obtain the prediction accuracy data through the SVR analysis (Table 1). Similar to the SVC analysis, classifier 3 achieved the highest accuracy (59.26\%) while the other 3 classifiers showed accuracies around $50 \%$ for the test dataset.

We also tried to use more stringent cut-off values for the classification basing on the SVR results. Again, higher prediction accuracies were achieved for the classifiers 2 and 3 , especially the latter (Table 3). For example, the accuracy is $100 \%$ for the test dataset if instances with predicted slope values $>1$ are classified into the price-rising up group and instances with values $<-0.2$ into the price-falling down group for classifier 3 (Figure 5). 


\section{Macrothink
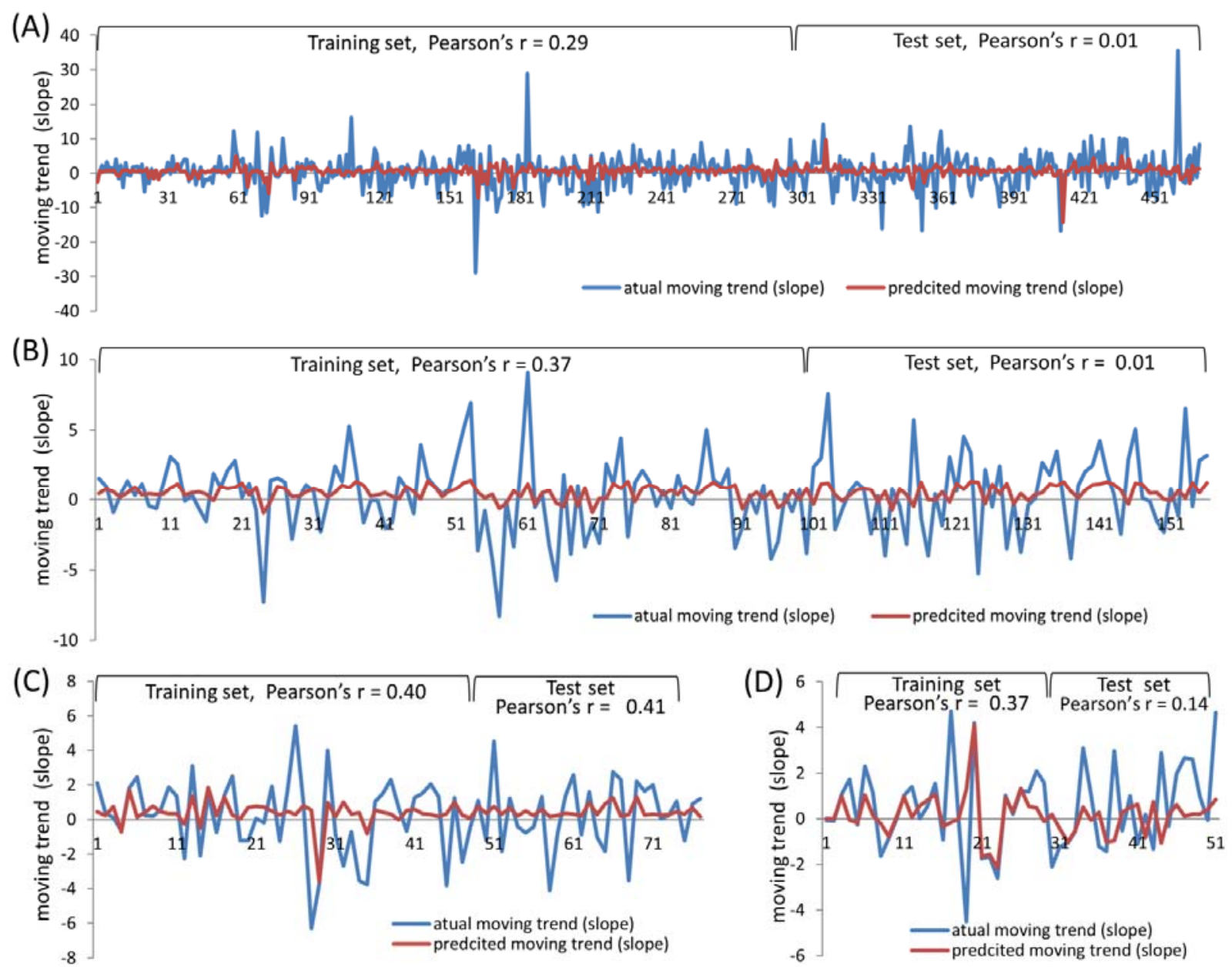

Figure 4. The correlation between the predicted and actual slope values across all the instances analyzed by SVR. (A) Classifier 1. (B) Classifier 2. (C) Classifier 3. (D) Classifier 4

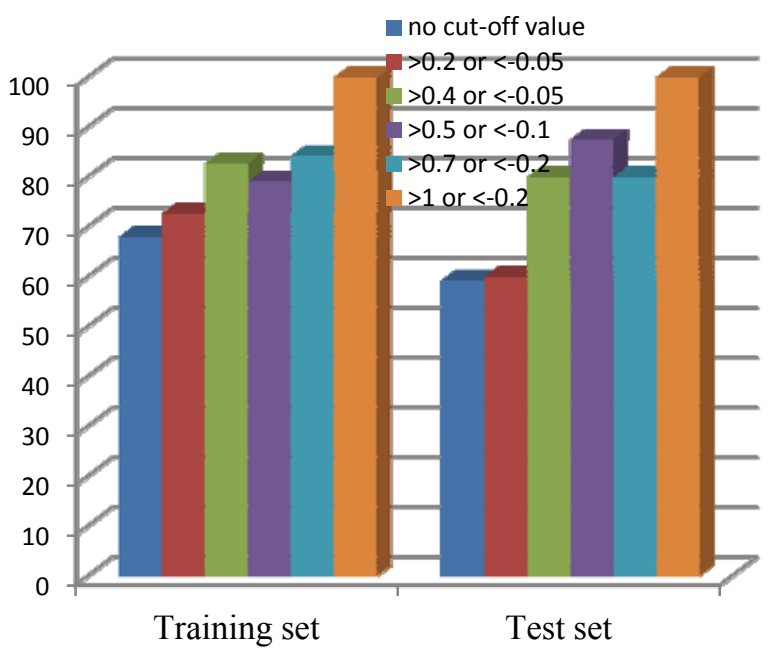

Figure 5. The prediction accuracies when using more stringent cut-off values for the classifier 3 basing on the SVR results 


\section{MInstitute ${ }^{\text {Macrothink }}$}
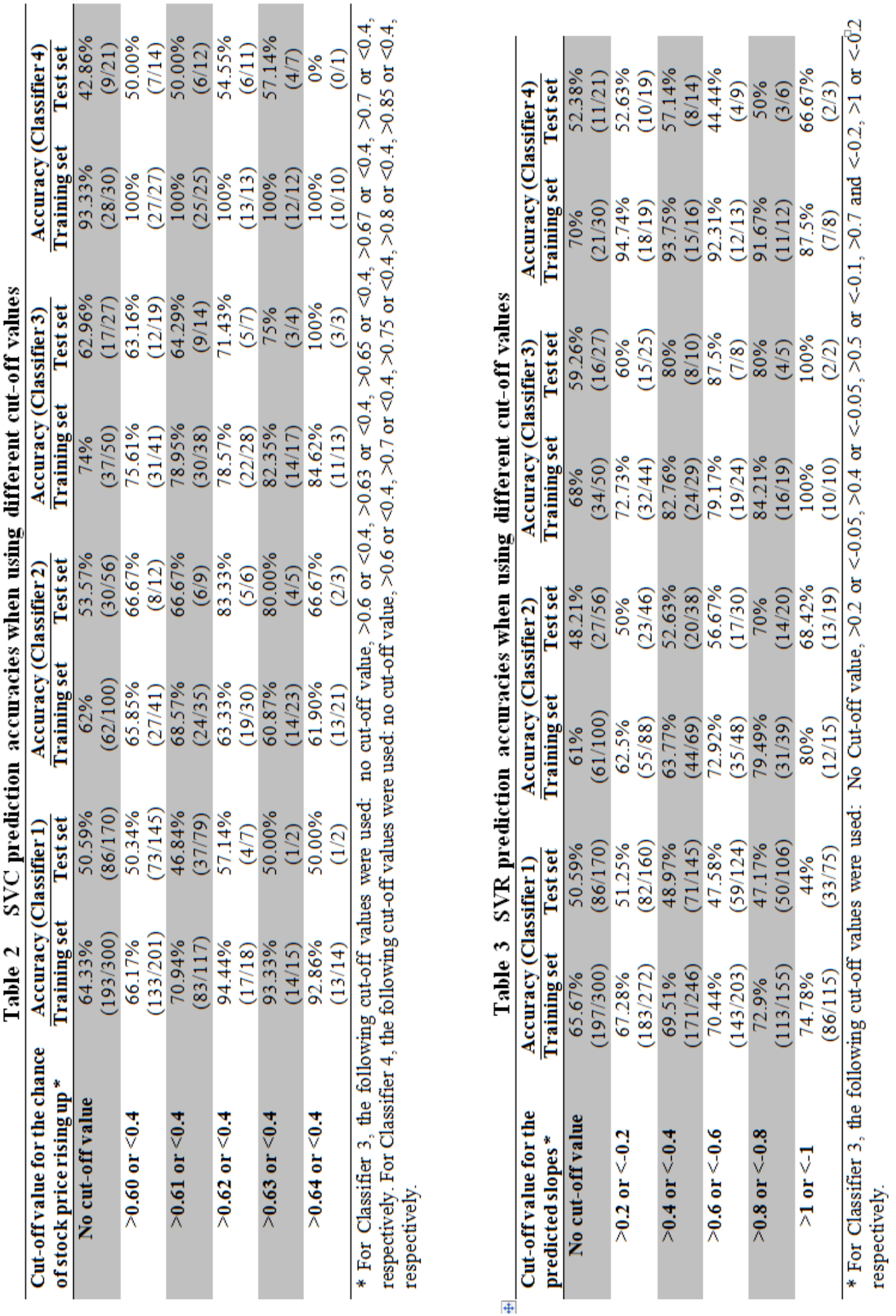


\subsection{Prediction of moving direction of GOOG price using SVC and SVR combination}

Figure 6 shows that combined use of SVC and SVR improves the prediction accuracy of the moving trend of GOOG stock price for classifier 2 and 3, especially the latter. We tested combinations at two levels. One is the combination of the classification results obtained when using the second cut-off value for SVC and SVR. The other one is the combination of the results when using the third cut-off vale for SVC and SVR. The principle for this combination is that only the instance classified into same group basing on SVC and SVR results are counted, and then the prediction accuracy are calculated basing on the percentage of instances being correctly classified by SVC and SVR.

(A)

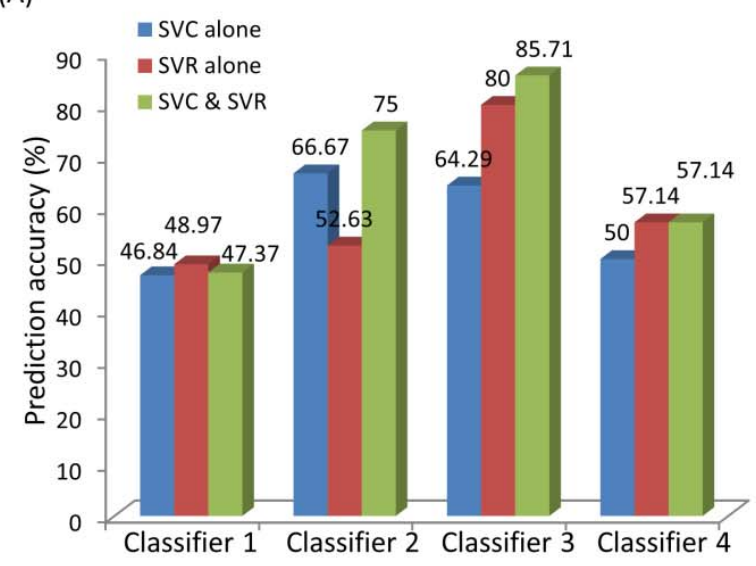

(B)

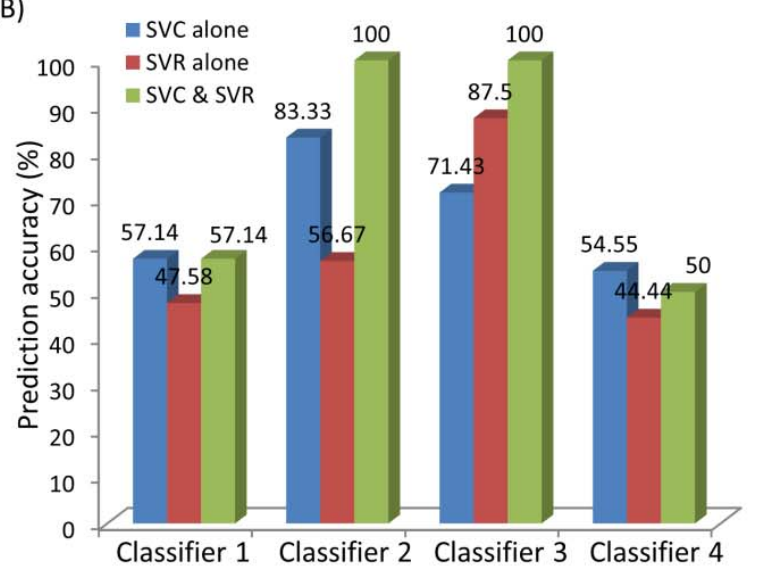

Figure 6. Combined using SVC and SVR improves the prediction accuracy for the moving trend of GOOG stock price. Columns represent the prediction accuracy for the test sets. (A) Combined analysis of the prediction results that were obtained using the second cut-off vale for SVC $(>0.61$ or $<0.4)$ and SVR $(>0.4$ or $<-0.4)$. (B) Combined results that were obtained using the third cut-off vale for SVC $(>0.62$ or $<0.4)$ and SVR $(>0.6$ or $<-0.6)$.

\section{Summary}

Using the stock of Google Inc. as an example, in this study we tested the potential application of the moving trends of the stock transaction data in the forecasting the movement direction of stock price with SVC and SVR methods. Our results indicate that the moving trends of transaction data within 30 trading days have the best prediction accuracy of the stock price in both methods, and combination of these two methods improves the prediction performance. The moving trends within short period are apt to be affected by various random events, which may explain our findings that moving trends within $\leq 15$ trading days failed to well predict the moving direction of the stock price. However, this does not mean that moving trends $>15$ trading days will always be good for stock price prediction, as we also found that the trends within 45 trading days had the similar prediction performance as that within 15 trading days. Prediction using the trends within $>45$ trading days (such as 60 and 75 trading days) failed to improve the performance either (data not shown). These results indicated that moving trends 
of stock transaction data within a certain time period have good inertia and are thus useful for forecasting the moving direction of stock price.

\section{References}

Abu-Mostafa, Y. S., \& Atiya, A. F. (1996). Introduction to financial forecasting. Applied Intelligence, 6, 205-213. http://dx.doi.org/10.1007/BF00126626

Chang, C. and Lin C. (2011). LIBSVM : a library for support vector machines. $A C M$ Transactions on Intelligent Systems and Technology, 2, 1-27. http://dx.doi.org/10.1145/1961189.1961199

Chen, Wun Hua, Shih, Jen Ying, \& Wu, Soushan. (2006). Comparison of support-vector machines and back propagation neural networks in forecasting the six major Asian stock markets. International Journal of Electronic Finance, 1, 49-67. http://dx.doi.org/10.1504/IJEF.2006.008837

Corwin, Shane A. and Schultz, Paul. (2012). A Simple Way to Estimate Bid-Ask Spreads from Daily High and Low Prices. The Journal of Finance, 67, 719-760. http://dx.doi.org/10.1111/j.1540-6261.2012.01729.x

Fiess, Norbert M., \& MacDonald, Ronald. (2002). Towards the fundamentals of technical analysis: analysing the information content of High, Low and Close prices. Economic Modelling, 19 , http://www.sciencedirect.com/science/article/pii/S0264999301000670

353-374.

Fuertes, Ana Maria, \& Olmo, Jose. (2013). Optimally harnessing inter-day and intra-day information for daily value-at-risk prediction. International Journal of Forecasting, 29, 28-42. http://dx.doi.org/10.2139/ssrn.1924237

Grosan C, \& Abraham A. (2006) Stock Market Modeling Using Genetic Programming Ensembles. In: Nedjah N, Mourelle L, Abraham A, editors. Genetic Systems Programming. 13 ed., 131-46. Springer Berlin Heidelberg. http://dx.doi.org/10.1007/3-540-32498-4_6

Hsu C-W, Chang C-C, \& Lin C-J. (2005) A Practical Guide to Support Vector Classication. http://www.csie.ntu.edu.tw/ cjlin.

Kao, Ling Jing, Chiu, Chih Chou, Lu, Chi Jie, \& Yang, Jung Li. (2013). Integration of nonlinear independent component analysis and support vector regression for stock price forecasting. Neurocomputing, 99, 534-542. http://dx.doi.org/10.1016/j.neucom.2012.06.037

Kazem, Ahmad, Sharifi, Ebrahim, Hussain, Farookh Khadeer, Saberi, Morteza, \& Hussain, Omar Khadeer. (2013). Support vector regression with chaos-based firefly algorithm for stock market price forecasting. Applied Soft Computing, 13, 947-958. http://dx.doi.org/10.1016/j.asoc.2012.09.024

Lildholdt P. (2002). Estimation of GARCH models based on open, close, high, and low prices. CAF, Centre for Analytical Finance.

Murphy J. J. (1999). Technical analysis of the financial markets: a comprehensive guide to trading methods and applications, 2. Prentice Hall Press.

Sapankevych, N. I., \& Sankar, Ravi. (2009). Time Series Prediction Using Support Vector Machines: A Survey. Computational Intelligence Magazine, IEEE, 4, 24-38. http://dx.doi.org/10.1109/MCI.2009.932254 


\section{Macrothink}

Asian Journal of Finance \& Accounting ISSN 1946-052X

Turner T. (2007). A Beginner's Guide to Day Trading Online. Adams Media, 2nd edition.

Vapnik V. (1995). The Nature of Statistical Learning Theory. Springer, N.Y., ISBN 0-387-94559-8.

Yang H, Chan L, \& King I. (2002). Support Vector Machine Regression for Volatile Stock Market Prediction. In: Yin H, Allinson N, Freeman R, Keane J, Hubbard S, editors. Intelligent Data Engineering and Automated Learning-IDEAL 2002. 2412 ed. Springer Berlin Heidelberg; p. 391-6.

Yoo PD, Kim MH, \& Jan T. (2005). Proceedings of the 2005 International Conference on Computational Intelligence for Modelling, Control and Automation, and International Conference on Intelligent Agents, Web Technologies and Internet Commerce (CIMCA-IAWTIC'05).

Zhi-gang, Wang, Chi-she, Wang, Qing-xia, Ma, \& Yong, Hu. (2013). The securities market month K line forecast based on SVC. Information Science and Management Engineering (Set), 46, 235-243. http://dx.doi.org/10.2495/ISME20130311 